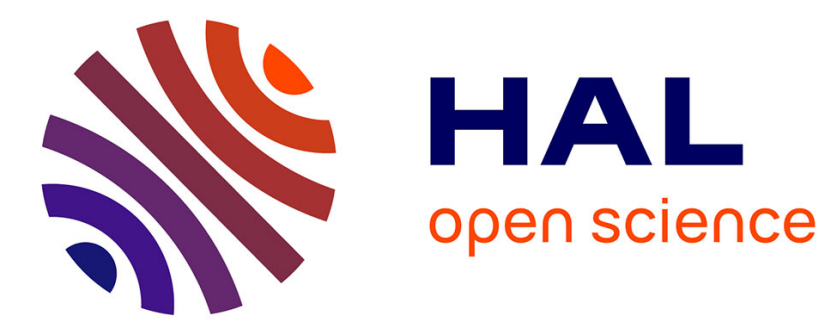

\title{
INTERACTION OF SOLITONS FROM THE PDE POINT OF VIEW
}

Yvan Martel

\section{To cite this version:}

Yvan Martel. INTERACTION OF SOLITONS FROM THE PDE POINT OF VIEW. 2018. hal01856447

\section{HAL Id: hal-01856447 \\ https://hal.science/hal-01856447}

Preprint submitted on 11 Aug 2018

HAL is a multi-disciplinary open access archive for the deposit and dissemination of scientific research documents, whether they are published or not. The documents may come from teaching and research institutions in France or abroad, or from public or private research centers.
L'archive ouverte pluridisciplinaire HAL, est destinée au dépôt et à la diffusion de documents scientifiques de niveau recherche, publiés ou non, émanant des établissements d'enseignement et de recherche français ou étrangers, des laboratoires publics ou privés. 


\title{
INTERACTION OF SOLITONS FROM THE PDE POINT OF VIEW
}

\author{
YVAN MARTEL
}

\begin{abstract}
We review recent results concerning the interactions of solitary waves for several universal nonlinear dispersive or wave equations. Though using quite different techniques, these results are partly inspired by classical papers based on the inverse scattering theory for integrable models.
\end{abstract}

\section{INTRODUCTION}

Pioneering numerical experiments of Fermi, Pasta, Ulam [49] in 1955, and of Zabusky, Kruskal [149] in 1965, revealed unexpected phenomena related to the interactions of nonlinear waves ${ }^{1}$. Shortly thereafter, the inverse scattering theory and its generalizations, developed by many influential mathematicians $[1,50,51,92,114,115,151]$, provided a rigorous ground and a unified approach to these observations. It led very rapidly to an accurate and deep understanding of remarkable properties of several universal nonlinear models, referred to as completely integrable, such as for example, the Korteweg-de Vries equation, the one dimensional cubic Schrödinger equation and the sine-Gordon equation. It has created a very active and inspiring field of research since then ${ }^{2}$. Among the most notable achievements of this theory, we mention

(i) the existence of infinitely many conservation laws;

(ii) the purely elastic nature of the collision of any number of solitary waves, which means that the interacting solitary waves recover their exact shape and velocity after a collision. Solitary waves enjoying such remarkable property were called solitons ${ }^{3}$;

(iii) the decomposition into solitons, saying that from any solution should emerge in large time a sum of nonlinear states, such as solitons, plus a dispersive part.

These rigorous mathematical facts are known to be physically relevant in numerous contexts, though sometimes under less extreme forms. For example, in several practical applications or for more elaborate nonlinear models, the collision of nonlinear waves is not purely elastic and some loss of energy takes place during collisions ${ }^{4}$. This reveals that the inverse scattering theory is restricted to models with specific algebraic structure and despite many extensions to nearly integrable systems (see e.g. [79]), it cannot be applied to general nonlinear models.

2010 Mathematics Subject Classification. Primary: 35B40; Secondary: 37K40,35Q51,35Q53,35Q55,35L71.

Key words and phrases. soliton, multi-soliton, asymptotic behavior of solutions, elastic and non-elastic collisions, nonlinear Schrödinger equation, generalized Korteweg-de Vries equation, semilinear wave equation.

${ }^{1}$ We refer to Chapter 8 of [34] for details on this discovery and on the relation between the model considered in [49] and the $\mathrm{KdV}$ equation. It is quite rightly suggested in [34] to recognize the work of M. Tsingou, contributor to the numerical computations of [49].

${ }^{2}$ See for example [91] and Chapter 7 of [34] for synthetic presentations of the inverse scattering transform.

${ }^{3}$ This term is now commonly used for solitary waves even in the non-integrable context.

${ }^{4}$ We refer to [29] for a discussion on this topic. 
In view of the beautiful achievements of the integrability theory but also of its inevitable limitations, it appeared necessary to investigate similar questions for general nonlinear models with solitary waves using tools from the theory of partial differential equations. In these notes, we review some results on interactions of solitary waves obtained for models that are not close to any known integrable equation, such as the generalized Korteweg-de Vries equation, the nonlinear Schrödinger equation in any space dimension, the $\phi^{4}$ equation and the nonlinear wave equation.

Mainly in the 80s, the solitary wave theory, proving existence, uniqueness, symmetry and stability properties of nonlinear waves, was successfully developed using the elliptic theory, ODE analysis and general variational arguments, at least for ground states (see Section 3). More recently, asymptotic stability results appeared (see Section 4). Then, energy type arguments extending the elliptic theory have allowed to consider several solitary waves in weak interactions, i.e. cases where the soliton dynamics is only slightly perturbed by the interactions. Pushing the perturbative analysis one step forward, some examples of strong interactions have also been exhibited; the solitons are still distant, but their dynamics is substantially modified by the interactions (see Section 5). Next, we review the few recent cases where a version of the soliton resolution conjecture was proved for non-integrable wave models in Section 6 . Finally, we discuss in Section 7 some situations where collisions were proved to be inelastic.

This review points out that despite some impressive and surprizing recent progress, notably on the soliton resolution conjecture, most of the questions raised above on the interaction of solitary waves remain open for general nonlinear models.

\section{INTEGRABLE EQUATIONS}

In this section, we briefly highlight some results from the inverse scattering theory that inspired mathematical research much beyond their range of applicability.

2.1. KdV solitons and multi-solitons. For the Korteweg-de Vries equation ${ }^{5}$

$$
\partial_{t} u+\partial_{x}\left(\partial_{x}^{2} u+u^{2}\right)=0, \quad(t, x) \in \mathbb{R} \times \mathbb{R},
$$

the inverse scattering transform led to a very striking property which is the existence of exact multi-soliton solutions (see e.g. [63, 114, 147]).

Let $Q(x)=\frac{3}{2} \cosh ^{-2}\left(\frac{x}{2}\right)$ be the unique positive even solution of $Q^{\prime \prime}+Q^{2}=Q$, and for $c>0$, let $Q_{c}(x)=c Q(\sqrt{c} x)$. Then, for any $c>0, \sigma \in \mathbb{R}$, the function defined by $u(t, x)=$ $Q_{c}(x-c t-\sigma)$ is a solution of (1), called soliton, traveling with speed $c$.

Solutions containing an arbitrary number of such solitons (called multi-solitons) have been obtained by the inverse scattering theory.

Theorem 1 (Multi-solitons for $\mathrm{KdV},[63,114]$ ). Let $K \in \mathbb{N}, K \geq 2$. Let $0<c_{K}<\cdots<c_{1}$ and $\sigma_{1}^{-}, \ldots, \sigma_{K}^{-} \in \mathbb{R}$. There exist $\sigma_{1}^{+}, \ldots, \sigma_{K}^{+} \in \mathbb{R}$ and an explicit solution $u$ of (1) such that

$$
\lim _{t \rightarrow \pm \infty}\left\|u(t)-\sum_{k=1}^{K} Q_{c_{k}}\left(\cdot-c_{k} t-\sigma_{k}^{ \pm}\right)\right\|_{H^{1}}=0 .
$$

The most remarkable fact is that all the solitons recover exactly the same sizes and speeds after the collision. Moreover, the values of $\sigma_{k}^{+}$are explicit. It it interesting to recall that the multi-soliton behavior, even in the simple case of two solitons, differs qualitatively according to the relative sizes of the solitons. We refer to Lemma 2.3 in [92] for a definition of the three

\footnotetext{
${ }^{5}$ We refer to Chapter 1 of [34] for historical facts on this equation and its applications to Physics.
} 
Lax categories of two-solitons and to [149] for a previous formal discussion. In particular, if their sizes are close (i.e. $c_{1} \sim c_{2}$ ), the two solitons never cross, but rather repulse each other at a large distance (this is category (c) in [92]). See $§ 7.1-7.3$.

2.2. Decomposition into solitons for $\mathbf{K d V}$. The multi-soliton behavior is fundamental for general solutions of the KdV equation as shown by the following decomposition result.

Theorem 2 (Decomposition into solitons, $[46,134]$ ). Let $u_{0}$ be a $C^{4}$ function such that for any $j \in\{0, \ldots, 4\}$, for all $x \in \mathbb{R},\left|\left(\partial^{j} u_{0} / \partial x^{j}\right)(x)\right| \lesssim\langle x\rangle^{-10}$. Let $u$ be the solution of $(1)$ corresponding to $u_{0}$. Then, there exist $K \in \mathbb{N}, \sigma_{1}, \ldots, \sigma_{K} \in \mathbb{R}$ and $c_{1}>\cdots>c_{K}>0$ such that, for all $x>0$,

$$
\lim _{t \rightarrow+\infty}\left\{u(t, x)-\sum_{k=1}^{K} Q_{c_{k}}\left(x-c_{k} t-\sigma_{k}\right)\right\}=0 .
$$

This result has a rich history, see $[1,17,34,39,46,88,92,134,149]$ and the references therein. Note that if some space decay is necessary to apply the inverse scattering transform, the decay assumption on the initial data in the above result is not optimal. Note also that the asymptotic behavior of the solution is described for $x>0$ (see results in [134] for slight improvement). For the region $x<0$, see [36, 46, 134] and references therein.

Last, we mention that the modified $\mathrm{KdV}$ equation (i.e. the $\mathrm{KdV}$ equation with a cubic nonlinearity) is also an integrable model that enjoys most of the properties of the $\mathrm{KdV}$ equation, like the infinitely many conservation laws and the existence of pure multi-soliton solutions (see e.g. [114]). Actually, it even has a richer family of exceptional solutions: breather solutions (see [3, 91, 142]) and dipole solitons, i.e. special multi-solitons where solitons are distant like $C \log t$ (see $[56,72,143]$ ). This complicates any possible soliton resolution conjecture on this equation (see [134]).

2.3. One dimensional cubic NLS. The 1D cubic nonlinear Schrödinger equation

$$
i \partial_{t} u+\partial_{x}^{2} u+|u|^{2} u=0, \quad(t, x) \in \mathbb{R} \times \mathbb{R},
$$

is also an integrable equation, widely studied for its numerous physical applications and remarkable mathematical properties. See e.g. [34, 35, 37, 39, 48, 121, 124, 148, 149, 150, 151].

Here, we denote $Q(x)=\sqrt{2} \cosh ^{-1}(x)$ the unique positive even solution of $Q^{\prime \prime}+Q^{3}=Q$, and for any $c>0, Q_{\lambda}(x)=\sqrt{c} Q(\sqrt{c} x)$. Then, for any $c>0, \beta \in \mathbb{R}, \sigma \in \mathbb{R}$ and $\gamma \in \mathbb{R}$,

$$
u(t, x)=Q_{c}(x-\beta t-\sigma) e^{i \Gamma(t, x)}, \quad \Gamma(t, x)=\frac{1}{2}(\beta \cdot x)-\frac{1}{4}|\beta|^{2} t+c t+\gamma,
$$

is a solitary wave of (2), moving on the line $x=\sigma+\beta t$ and also called soliton.

As the KdV equation, the 1D cubic NLS admits explicit multi-solitons. However, the possible behaviors of multi-solitons is richer for NLS. In addition to multi-solitons distant like $C t$, which is the generic situation, the equation also admits multi-solitons where the distance between some solitons is $C \log t$ (see [124, 151]; this requires solitons of exactly the same size, like for $\mathrm{mKdV}$ ) and solutions where some solitons are staying at a finite distance from each other for all time (see $[148,151]$ ). As for $\mathrm{mKdV}$, the presence of such multi-solitons complicates any general decomposition result but does not prevent it. For such questions, we refer to the recent work [11] and its references. 
2.4. The sine-Gordon equation. The sine-Gordon equation

$$
\partial_{t}^{2} u-\partial_{x}^{2} u+\sin u=0, \quad(t, x) \in \mathbb{R} \times \mathbb{R},
$$

was also widely studied as a physically relevant and completely integrable model (see e.g. [34, $39,91])$. This equation has an explicit kink solution $S(x)=4 \arctan \left(e^{x}\right)$. It also has other exceptional solutions, like time-periodic wobbling kinks (see [33, 135]), and breathers (see [91]).

2.5. Other integrable models and nearly integrable models. For the derivative NLS equation, we refer to [71] and its references. For the KP-I equation, see Lamb [91]. For integrable models set on the torus, see [89] and references therein.

Several nearly integrable equations have also been studied in the context of the theory of inverse scattering. We refer to $[34,38,79,148]$ and to the references therein.

2.6. Formal works and numerical simulations. Note that shortly after the development of the inverse scattering and the discovery of explicit multi-solitons, other approaches appeared, like in $[47,56,72]$, to investigate possible multi-soliton behaviors for integrable or non-integrable models. Such papers focus on the modulation equations of the parameters of the solitons and lack the analysis of the error terms, but they aim at justifying formally multisolitons behaviors beyond any integrability property or proximity to integrable equations. In particular, as for the rigorous results presented in Section 5 below, they are asymptotic results, restricted to cases where the distances between the various solitons are large enough.

Theoretical and numerical works have been developed in parallel. As mentioned in the Introduction, the subject started with two fundamental numerical experiments presented in $[49,149]$. Since then, there has been an intense activity on studying solitary waves interactions from the numerical point of view. We refer to [29] which compares KdV multi-solitons, the water wave problem from the numerical point of view and real experiments on waves generated in water tanks. For Klein-Gordon equations, we refer to [2]. We also refer to $[9,34,59,94,136,148]$ and references therein. One of the main questions studied by numerical experiments is the elastic versus inelastic character of the collisions of nonlinear waves.

\section{NONLinear MOdels With SOlitary WAVES}

In these notes, we consider four typical nonlinear models and work with the notion of solution in the energy space.

3.1. The generalized Korteweg-de Vries equation. Consider the following 1D model, for any integer $p \geq 2$,

$$
\partial_{t} u+\partial_{x}\left(\partial_{x}^{2} u+u^{p}\right)=0, \quad(t, x) \in \mathbb{R} \times \mathbb{R} .
$$

As seen before, the case $p=2$ corresponds to the $\mathrm{KdV}$ equation and $p=3$ to the $\mathrm{mKdV}$ equation, which are both completely integrable.

The mass and energy

$$
\int u^{2}(t), \quad \int\left(\frac{1}{2} u_{x}^{2}(t)-\frac{1}{p+1} u^{p+1}(t)\right)
$$

are formally conserved for solutions of (3). We refer to [78] for the local well-posedness of the Cauchy problem in the energy space $H^{1}$ (see also [73]). For $1<p<5$, all solutions in $H^{1}$ are global and bounded, and the problem is called sub-critical. For $p=5$, the problem is mass critical (blow up solution do exist, see [107] and references therein) and $p>5$ correspond to the super-critical case. The notion of criticality corresponds to the scaling invariance of 
equation (3): indeed, if $u(t, x)$ is solution then for any $c>0, u_{c}(t, x)=c^{\frac{1}{p-1}} u\left(c^{\frac{3}{2}} t, c^{\frac{1}{2}} x\right)$ is also solution and $\left\|u_{c}(t)\right\|_{L^{2}}=c^{\frac{1}{p-1}-\frac{1}{4}}\|u(t)\|_{L^{2}}$.

Let $Q$ be the unique (up to sign change if $p$ is odd) non-trivial even solution of $Q^{\prime \prime}+Q^{p}=Q$ on $\mathbb{R}$, explicitely given by

$$
Q(x)=\left(\frac{p+1}{2}\right)^{\frac{1}{p-1}} \cosh ^{-\frac{2}{p-1}}\left(\frac{p-1}{2} x\right) .
$$

For $c>0$, let $Q_{c}(x)=c^{\frac{1}{p-1}} Q\left(c^{\frac{1}{2}} x\right)$. Note that these formulas for $Q$ and $Q_{c}$ generalize the previous ones given for $p=2$ and $p=3$. As before, solitary waves (also called solitons by abuse of terminology) are solutions of (3) of the form $u(t, x)=Q_{c}(x-c t-\sigma)$, for any $c>0$ and $\sigma \in \mathbb{R}$.

The orbital stability of solitons with respect to small perturbations in the energy space $H^{1}$ is known in the sub-critical case.

Theorem 3 (Stability of the soliton for sub-critical gKdV $[6,8,16,145,146]$ ). Let $1<p<5$. For all $\epsilon>0$, there exists $\delta>0$, such that if $\left\|u_{0}-Q\right\|_{H^{1}} \leq \delta$, then the solution $u$ of (3) with initial data $u_{0}$ satisfies, for all $t \in \mathbb{R},\|u(t, .+\sigma(t))-Q\|_{H^{1}} \leq \epsilon$, for some function $\sigma$.

In contrast, solitons are unstable in the critical and super-critical case $p \geq 5$. Note that the instability phenomenon is quite different in the critical case (linear stability holds and the nonlinear instability is related to the scaling parameter) and in the super-critical case (linear exponential instability). See $[10,15,58,97,125]$.

3.2. The nonlinear Schrödinger equation. Recall the nonlinear NLS equation

$$
i \partial_{t} u-\Delta u-|u|^{p-1} u=0, \quad(t, x) \in \mathbb{R} \times \mathbb{R}^{d} .
$$

We consider the case $p>1$ for $d=1,2$, and $1<p<\frac{d+2}{d-2}$ for $d \geq 3$. For $d=1$ and $p=3$, the model is completely integrable, as seen before. Note that, similarly as for the gKdV equation, $p=1+\frac{4}{d}$ corresponds to $L^{2}$ criticality, while for $d \geq 3, p=\frac{d+2}{d-2}$ corresponds to $\dot{H}^{1}$ criticality.

The mass, energy and momentum

$$
\int|u(t)|^{2}, \quad \int\left(\frac{1}{2}|\nabla u(t)|^{2}-\frac{1}{p+1}|u(t)|^{p+1}\right), \quad \Im\left(\int \nabla u(t) \bar{u}(t)\right)
$$

are formally conserved for solutions of $(4)$. We refer to $[15,55,139]$ for the local well-posedness of the Cauchy problem in the energy space $H^{1}$.

We denote by $Q$ the unique positive radially symmetric $H^{1}$ solution of $\Delta Q+|Q|^{p-1} Q=Q$ on $\mathbb{R}^{d}$ (the function $Q$ is called the ground state; see existence and uniqueness results in $[7,15,90,139])$. For $c>0$, let $Q_{c}(x)=c^{\frac{1}{p-1}} Q\left(c^{\frac{1}{2}} x\right)$. Note that this is a further generalization of the notation for $\mathrm{gKdV}$, for any space dimension $d \geq 1$. For $d \geq 2$, ground states are no longer explicit, but their properties are well-understood (see references above). Then, for any $c>0, \beta \in \mathbb{R}^{d}, \sigma \in \mathbb{R}^{d}$ and $\gamma \in \mathbb{R}$, the function $u$ defined by

$$
u(t, x)=Q_{c}(x-\beta t-\sigma) e^{i \Gamma(t, x)} \quad \text { where } \quad \Gamma(t, x)=\frac{1}{2}(\beta \cdot x)-\frac{1}{4}|\beta|^{2} t+c t+\gamma,
$$

is a traveling wave of (4), with speed $\beta$.

The stability and instability properties of solitary waves of NLS are similar: stability in the $L^{2}$ sub-critical case, and instability in the critical and super-critical cases. We refer to $[15,16,57,58,145]$ for details. 
3.3. The $\phi^{4}$ equation. We consider the $\phi^{4}$ model (see e.g. [34, 95])

$$
\partial_{t}^{2} \phi-\partial_{x}^{2} \phi-\phi+\phi^{3}=0, \quad(t, x) \in \mathbb{R} \times \mathbb{R} .
$$

Recall that the energy

$$
E\left(\phi, \partial_{t} \phi\right)=\int \frac{1}{2}\left|\partial_{t} \phi\right|^{2}+\frac{1}{2}\left|\partial_{x} \phi\right|^{2}+\frac{1}{4}\left(1-|\phi|^{2}\right)^{2}
$$

is formally conserved along the flow. The kink, defined by $H(x)=\tanh (x / \sqrt{2})$ is the unique (up to sign change), bounded, odd solution of the equation $-H^{\prime \prime}=H-H^{3}$ on $\mathbb{R}$. We recall that the orbital stability of the kink with respect to small perturbations in the energy space has been proved in [62] using mainly the energy conservation. This model is analogue to the sine-Gordon equation, but it is not completely integrable and breathers solutions or woobling kinks are not expected to exist.

3.4. The energy critical nonlinear wave equation. For space dimensions $d \geq 3$, we consider the following nonlinear wave equation,

$$
\partial_{t}^{2} u=\Delta u+|u|^{\frac{4}{d-2}} u, \quad(t, x) \in \mathbb{R} \times \mathbb{R}^{d} .
$$

We denote

$$
E(u, v)=\int\left(\frac{1}{2}|\nabla u|^{2}+\frac{1}{2} v^{2}-\frac{1}{6}|u|^{6}\right)
$$

so that the energy of a solution $\left(u, \partial_{t} u\right)$ of $(6)$, defined by $E\left(u, \partial_{t} u\right)$, is formally conserved by the flow. Concerning the Cauchy problem in $\dot{H}^{1} \times L^{2}$ for the energy critical wave equation, we refer to [77] and the references given therein. As before, the notion of criticality is related to the scaling invariance: if $u(t, x)$ is a solution, then for any $\lambda>0$,

$$
u_{\lambda}(t, x)=\frac{1}{\lambda^{\frac{d-2}{2}}} u\left(\frac{t}{\lambda}, \frac{x}{\lambda}\right) \quad \text { is also solution and }\left\|\nabla u_{\lambda}\right\|_{L^{2}}=\|\nabla u\|_{L^{2}} .
$$

Here, solitary waves are stationary solutions $W \in \dot{H}^{1}$ satisfying $\Delta W+|W|^{\frac{4}{d-2}} W=0$, and traveling waves obtained as Lorentz transforms of such solutions. For $\ell \in \mathbb{R}^{d},|\ell|<1$, we denote

$$
W_{\ell}(x)=W\left(\left(\frac{1}{\sqrt{1-|\ell|^{2}}}-1\right) \frac{\ell(\ell \cdot x)}{|\ell|^{2}}+x\right),
$$

so that $u(t, x)=W_{\ell}(x-\ell t)$ is solution of (6). As for the NLS equation, we consider only ground states solitary waves, i.e. solutions of the above elliptic equation explicitely given by

$$
W(x)=\left(1+\frac{|x|^{2}}{d(d-2)}\right)^{-\frac{d-2}{2}} .
$$

As solutions of the evolution equation (6), they are unstable with respect to perturbation of the initial data with one direction of exponential instability (see $[40,57]$ ).

\section{Asymptotic stability}

We recall briefly some results of asymptotic stability of solitons. 


\subsection{Asymptotic stability for gKdV solitons.}

Theorem 4 (Asymptotic stability of the gKdV soliton in $H^{1}$, [98]). Let $p=2,3,4$. For any $\beta>0$, there exists $\delta=\delta(\beta)>0$ such that the following is true. Let $u_{0} \in H^{1}$ be such that $\left\|u_{0}-Q\right\|_{H^{1}} \leq \delta$. Then, the global solution $u$ of (3) with initial data $u_{0}$ satisfies

$$
\lim _{t \rightarrow+\infty}\left\|u(t)-Q_{c^{+}}(\cdot-\sigma(t))\right\|_{H^{1}(x>\beta t)}=0,
$$

for some $c^{+}>0$ with $\left|c^{+}-1\right| \lesssim \delta$ and some $C^{1}$ function $\sigma$ such that $\lim _{+\infty} \sigma^{\prime}=c^{+}$.

We refer to [126] for the first result of asymptotic stability of gKdV solitons. Theorem 1 claims strong convergence in $H^{1}$ in the region $x>\beta t$. Strong convergence in $H^{1}(\mathbb{R})$ is never true since it would imply by stability that $u$ is exactly a soliton. The region where convergence is obtained in Theorem 1 is sharp since one can construct a solution which behaves asymptotically as $t \rightarrow+\infty$ as the sum $Q(x-t)+Q_{c}(x-c t)$, where $0<c \neq 1$ is arbitrary (see $[96,114,144]$ ). In particular, choosing $c \ll 1$, the $H^{1}$ norm of $Q_{c}(x-c t)$ is small, and this soliton travels on the line $x=c t$. This explains the necessity of a positive $\beta$ in the convergence result. We also refer to the survey [141]. For $p=4$, the result has been completed in $[76,140]$ showing that the residue scatters in a Besov space close to the critical Sobolev space $\dot{H}^{-1 / 6}$. For $p=3$, we refer to [54] for a full asymptotic stability statement.

4.2. Asymptotic stability for NLS equations. In the context of the nonlinear Schrödinger equation, pioneering results on asymptotic stability of traveling waves are [12, 13, 137, 138]. These papers initiated the method of separating modes and using dispersive estimates with potential, under assumptions on the spectrum of the linearized operator.

This question has then been extensively studied, for the NLS equation with or without potential and for various nonlinearities, see e.g. [14, 31, 53, 120, 129, 130, 131, 132, 133] as typical papers, and their references. Most of these works require specific assumptions, like spectral assumptions or suitable dispersive estimates for equations with unknown potential, a suitable Fermi Golden Rule or flatness conditions on the nonlinearities at 0. It follows that no result of asymptotic stability is fully proved for any pure power NLS equation without potential with stable solitons, except for the integrable cubic 1D NLS treated in [32].

In larger dimensions, or higher order nonlinearities, the solitons are unstable. The notion of conditional asymptotic stability and the construction of center stable manifolds then become relevant. For the focusing 3D cubic NLS equation (which is an $\dot{H}^{\frac{1}{2}}$ critical equation with exponentially unstable solitons) the theory has been especially well-developed, at least in the radial case, in $[4,5,22,120,131,133]$. In particular, spectral assumptions implying the desired dispersive estimates for the linearized equation around the soliton have been checked, first numerically and then rigorously by computer assisted proof (see [22] and references therein).

4.3. Asymptotic stability of the $\phi^{4}$ kink. The asymptotic stability of the kink $H$ by the $\phi^{4}$ flow (5) is known in the case of odd perturbations in the energy space. Note that for odd initial data, the corresponding solution of (5) is odd. Rewrite $\phi=H+u$. Then, one has

$$
\partial_{t}^{2} u-\partial_{x}^{2} u-u+3 H^{2} u+3 H u^{2}+u^{3}=0, \quad(t, x) \in \mathbb{R} \times \mathbb{R} .
$$

Theorem 5 (Asymptotic stability of the kink by odd perturbations, [82]). There exists $\delta>0$ such that for any odd $\left(u_{0}, u_{1}\right) \in H^{1} \times L^{2}$ with $\left\|\left(u_{0}, u_{1}\right)\right\|_{H^{1} \times L^{2}} \leq \delta$, the solution $\left(u, \partial_{t} u\right)$ of (7) with initial data $\left(u_{0}, u_{1}\right)$ satisfies, for any bounded interval $I \subset \mathbb{R}$,

$$
\lim _{t \rightarrow \pm \infty}\left\|\left(u, \partial_{t} u\right)(t)\right\|_{H^{1}(I) \times L^{2}(I)}=0 .
$$


As for $\mathrm{gKdV}$, if a solution $u$ of $(7)$ satisfies $\lim _{t \rightarrow+\infty}\left\|\left(u, \partial_{t} u\right)(t)\right\|_{H^{1} \times L^{2}}=0$, then by orbital stability [62], $u(t) \equiv 0$, for all $t \in \mathbb{R}$. Thus the local result is in some sense optimal.

For previous related results, we refer to $[80,81]$ where the asymptotic stability of the kink is studied for the $1 \mathrm{D}$ equation $\partial_{t}^{2} u-\partial_{x}^{2} u+F(u)=0$, under specific assumptions on $F$ (not including the $\phi^{4}$ model) and to [30], where the stability and asymptotic stability of the one dimensional kink for the $\phi^{4}$ model, subject to localized three dimensional perturbations is studied. We also refer the references in $[30,80,81,82]$ for related works on scattering of small solutions to Klein-Gordon equations. See also the review [83] and references therein.

4.4. Blow up profile for the critical wave equation. Recall that [77] provides a classification of all possible behaviors (blow up or scattering) of solutions of (6) whose initial data $\left(u_{0}, u_{1}\right)$ satisfies $E\left(u_{0}, u_{1}\right)<E(W, 0)$. Next, [40] studies the threshold case $E\left(u_{0}, u_{1}\right)=$ $E(W, 0)$ and constructs the stable manifold around $W$. Then, $[41,42]$ proved the following result for solutions slightly above the threshold.

Theorem 6 (Blow up profile for 3D critical NLW, [41, 42]). Let $d=3$. There exists $\delta>0$ such that if $u$ is a solution of (6) blowing up in finite time $T>0$ and satisfying the bound

$$
\sup _{[0, T)}\left(\|\nabla u(t)\|_{L^{2}}+\frac{1}{2}\left\|\partial_{t} u(t)\right\|_{L^{2}}\right) \leq\|\nabla W\|_{L^{2}}+\delta
$$

then

$$
\lim _{t \uparrow T}\left\|\left(u(t), \partial_{t} u(t)\right)-\left(v_{0}, v_{1}\right) \mp\left(\frac{1}{\lambda^{\frac{1}{2}}(t)} W_{\boldsymbol{\ell}}\left(\frac{\cdot-\sigma(t)}{\lambda(t)}\right),-\frac{1}{\lambda^{\frac{3}{2}}(t)}\left(\boldsymbol{\ell} \cdot \nabla W_{\boldsymbol{\ell}}\right)\left(\frac{\cdot-\sigma(t)}{\lambda(t)}\right)\right)\right\|_{L^{2}}=0
$$

for some $\sigma, \lambda$ and $\boldsymbol{\ell} \in \mathbb{R}^{3},|\boldsymbol{\ell}|<1$ and $\left(v_{0}, v_{1}\right) \in \dot{H}^{1} \times L^{2}$.

We see that the family $\left\{ \pm W_{\ell}\right\}$ is the universal blow up profile. We refer to the original papers for more results and details.

We refer to $[85,86]$ for classification results of solutions with energy at most slightly above the one of the ground state, and to $[87,68]$ for contructions of solutions with prescribed blow up rates (type II blow up). We also refer to $[99,107,111,112]$ and references therein for previous results of blow up profile in the case of mass critical gKdV and NLS equations. Concerning blow up, see also the review [128] and the references therein.

\section{AsYmptotic multi-SOLitons}

In this section, we discuss results of existence of asymptotic multi-solitons for non-integrable models, inspired by Theorem 1 and other explicit constructions of multi-solitons for integrable models, but limited to one direction of time. In particular, these results are valid in asymptotic situations where the distances between all the solitary waves are large enough.

5.1. Multi-solitons with weak interactions. As a rough idea, weak interaction means that the trajectories of the solitary waves are not affected asymptotically.

Theorem 7 (Existence and uniqueness of gKdV multi-solitons, [96]). Let $p=2,3$, 4 or 5 . Let $K \geq 2,0<c_{K}<\cdots<c_{1}$, and $\sigma_{1}, \ldots, \sigma_{K} \in \mathbb{R}$. Let $\mathbf{R}=\sum_{k=1}^{K} R_{k}$ where

$$
R_{k}(t, x)=Q_{c_{k}}\left(x-\sigma_{k}-c_{k} t\right) .
$$

There exists a unique $H^{1}$ solution of (3) such that $\lim _{t \rightarrow-\infty}\|u(t)-\mathbf{R}(t)\|_{H^{1}}=0$. Moreover, there exists $\kappa>0$ such that, for all $t \leq 0,\|u(t)-\mathbf{R}(t)\|_{H^{1}} \lesssim e^{-\kappa|t|}$. 
Such result shows that the multi-soliton behavior is universal, at least in one direction of time. Observe that it says nothing about the behavior the solution as $t \rightarrow+\infty$. The uniqueness statement in the energy space is relevant even in the integrable case since the inverse scattering theory requires a priori more space decay. The stability of the multi-soliton structure was studied in the sub-critical case in [108].

We observe that a similar existence result also holds for the gKdV super-critical equation $(p>5)$, with a specific classification related to the exponential instability, see $[18,27]$.

For the NLS equation, we recall the following existence result.

Theorem 8 (Existence of NLS multi-solitary waves, [27, 101, 110]). Let $d \geq 1$. Let $p>1$ for $d=1,2$ and $1<p<\frac{d+2}{d-2}$ for $d \geq 3$. Let $K \geq 2$ and for any $k \in\{1, \ldots, K\}$, let $c_{k}>0$, $\beta_{k} \in \mathbb{R}^{d}, \sigma_{k} \in \mathbb{R}^{d}$ and $\gamma_{k} \in \mathbb{R}$. Assume that, for any $k \neq k^{\prime}, \beta_{k} \neq \beta_{k^{\prime}}$. Let $\mathbf{R}=\sum_{k=1}^{K} R_{k}$ where

$$
R_{k}(t, x)=Q_{c_{k}}\left(x-\sigma_{k}-\beta_{k} t\right) e^{i \Gamma_{k}(t, x)} \quad \text { and } \quad \Gamma_{k}(t, x)=\frac{1}{2}\left(\beta_{k} \cdot x\right)-\frac{1}{4}\left|\beta_{k}\right|^{2} t+c_{k} t+\gamma_{k} .
$$

Then, there exist $T_{0} \in \mathbb{R}, \kappa>0$ and an $H^{1}$ solution $u$ of (4) such that, for all $t \leq T_{0}$, $\|u(t)-\mathbf{R}(t)\|_{H^{1}} \lesssim e^{-\kappa|t|}$.

Uniqueness (for critical and sub-critical nonlinearities) or classification (for super-critical nonlinearity) is an open problem. See [19] for multi-existence in the 1D super-critical case.

Note that the construction of multi-solitons and the study of the stability of the sums of multi-soliton has been extended to several other models, see e.g. $[26,28]$ for the case of the nonlinear Klein-Gordon equation, and [113] for the water wave model.

For the 5D energy critical wave equation, the following existence result is proved in [105].

Theorem 9 (Existence of NLW multi-solitary waves, [105]). Let $d=5$. Let $K \geq 2$, and for any $k \in\{1, \ldots, K\}$, let $\lambda_{k}>0, \boldsymbol{\sigma}_{k} \in \mathbb{R}^{5}, \iota_{k} \in\{-1,+1\}$ and $\boldsymbol{\ell}_{k} \in \mathbb{R}^{5}$ with $\left|\boldsymbol{\ell}_{k}\right|<1$. Assume that, for any $k^{\prime} \neq k, \boldsymbol{\ell}_{k} \neq \boldsymbol{\ell}_{k^{\prime}}$. Let $\mathbf{R}=\sum_{k=1}^{K} R_{k}$ where

$$
R_{k}(t, x)=\frac{\iota_{k}}{\lambda_{k}^{\frac{3}{2}}} W_{\boldsymbol{\ell}_{k}}\left(\frac{x-\boldsymbol{\ell}_{k} t-\boldsymbol{\sigma}_{k}}{\lambda_{k}}\right) .
$$

Assume that one of the following assumptions holds

(1) Two-solitons: $K=2$

(2) Collinear speeds: For all $k \in\{1, \ldots, K\}, \ell_{k}=\ell_{k} \mathbf{e}_{1}$ where $\ell_{k} \in(-1,1)$.

Then, there exist $T_{0} \in \mathbb{R}$ and a solution $u$ of $(6)$ on $\left(-\infty, T_{0}\right]$ in the energy space such that $\lim _{t \rightarrow-\infty}\left\|\nabla_{x, t}(u(t)-\mathbf{R}(t))\right\|_{L^{2}}=0$.

5.2. Multi-solitons with strong interactions. We state a typical result where the strong interactions between the traveling waves indeed affect their trajectories.

Theorem 10 (Two-solitary waves with logarithmic distance, [122]). Let $d \geq 1$. Let

$$
1<p<\frac{d+2}{d-2} \quad(p>1 \text { for } d=1,2) \quad \text { and } \quad p \neq 1+\frac{4}{d} .
$$

There exists a solution $u$ of (4) such that $\left|z_{1}(t)-z_{2}(t)\right| \sim 2 \log t$ as $t \rightarrow-\infty$ and

$$
\lim _{t \rightarrow-\infty}\left\|u(t)-e^{-i \gamma(t)} \sum_{k=1,2} Q\left(\cdot-z_{k}(t)\right)\right\|_{H^{1}}=0 .
$$


As discussed in $§ 2.3$, such solutions were already known in the integrable case by the inverse scattering theory. The above result means that this behavior is universal for general NLS equations, under the same restriction that the traveling waves have equal scaling. The mass critical case $p=1+\frac{4}{d}$ is excluded since it displays a special behavior related to blow up and where the above behavior is visible only in rescaled variables, as previously described in [109]. For the gKdV equation, a result similar to Theorem 10 is given in [123].

We mention a few other previous results of strong interactions: for the Hartree equation [84], for the energy critical wave equation [69, 70], for the mass critical gKdV equation [20, 21], and for the half-wave equation [52].

5.3. Soliton interaction with the background. Several papers deal with the question of the interaction of a soliton with a changing background or an impurity. See $[64,65,66,67]$ for the interaction of a soliton of NLS with a Dirac mass or a slowly varying potential, and $[118,119]$ for the interaction of a gKdV soliton with a slowing variable bottom.

\section{Decomposition into solitons For the EnERgy CRITiCAl WAVE EQUation}

Here, we recall the few existing results of decomposition in solitons in non-integrable cases. First, a complete result of decomposition into solitons for equation (6) was proved in [43] for the radial $3 \mathrm{D}$ case.

Theorem 11 (Soliton resolution for the 3D radial critical wave equation, [43]). Let $d=3$. Let $u$ be a global radial solution of (6). Then, there exist a solution $v_{\mathrm{L}}$ of the linear wave equation, $K \in \mathbb{N}, \iota_{k} \in\{-1,1\}, \lambda_{k}>0$, such that

$$
\lim _{t \rightarrow+\infty}\left\|\left(u(t), \partial_{t} u(t)\right)-\left(v_{\mathrm{L}}(t)+\sum_{k=1}^{K} \frac{\iota_{k}}{\lambda_{k}^{\frac{1}{2}}(t)} W\left(\frac{\cdot}{\lambda_{k}(t)}\right), \partial_{t} v_{\mathrm{L}}(t)\right)\right\|_{\dot{H}^{1} \times L^{2}}=0,
$$

and $\lambda_{1}(t) \ll \lambda_{2}(t) \ll \cdots \ll \lambda_{K}(t) \ll t$, as $t \rightarrow+\infty$.

Note that the above result is in some sense more complete than for $\operatorname{gKdV}(\S 2.1)$, since the residue is proved to scatter. A similar result holds for blow up solutions, provided they exhibit type II blow up. The soliton resolution conjecture was later proved in the non-radial case for a subsequence of time for the 3,4 and $5 \mathrm{D}$ energy critical wave equation in [44, 45]. Note that a fundamental idea in the approach of [43] is the introduction of the method of channels of energy for the linear wave equation (see Theorem 16 for a typical result in 5D).

See similar results for the wave map problem in [23, 24, 25].

\section{Collision problem}

Concerning the collision problem, we recall the discussion in [29] on inelastic collisions. To study the collision problem, it is natural to study the behavior as $t \rightarrow+\infty$ of the solutions constructed in Theorems 7, 8, 9. See [29], page 057106-4 for suggesting this approach which seems more canonical than to study initial data with the sum of two solitons initially distant.

7.1. Collision for the quartic $\mathbf{g K d V}$ equation $\mathbf{I}$. We consider the quartic gKdV equation

$$
\partial_{t} u+\partial_{x}\left(\partial_{x}^{2} u+u^{4}\right)=0 \quad(t, x) \in \mathbb{R} \times \mathbb{R} .
$$

The article [102] (see also [117] for generalization to any gKdV equation) gives the first rigorous results concerning collision of solitons for a non-integrable equation, and in particular the first 
proof of non-existence of pure two-soliton solutions, in the case where one soliton is much smaller than the other one.

Theorem 12 (Collision of solitons with very different size, [102]). Assume $0<c \ll 1$. Let $u$ be the solution of (8) such that

$$
\lim _{t \rightarrow-\infty}\left\|u(t)-Q(\cdot-t)-Q_{c}(\cdot-c t)\right\|_{H^{1}} \rightarrow 0 .
$$

(i) Global stability of the 2 -soliton behavior. There exist $c_{1}^{+} \underset{c \rightarrow 0}{\sim} 1, c_{2}^{+} \underset{c \rightarrow 0}{\sim} c, \rho_{1}, \rho_{2}$ such that the function $w^{+}$defined by

$$
w^{+}(t, x)=u(t, x)-Q_{c_{1}^{+}}\left(x-\rho_{1}(t)\right)-Q_{c_{2}^{+}}\left(x-\rho_{2}(t)\right)
$$

satisfies

$$
\lim _{t \rightarrow+\infty}\left\|w^{+}(t)\right\|_{H^{1}\left(x \geq \frac{c}{10} t\right)}=0 \quad \text { and } \quad \sup _{t \in \mathbb{R}}\left\|w^{+}(t)\right\|_{H^{1}} \lesssim c^{\frac{1}{3}} .
$$

(ii) Inelasticity of the collision. Moreover, for $t \gg 1$,

$$
c_{1}^{+}-1 \gtrsim c^{\frac{17}{6}}, \quad 1-\frac{c_{2}^{+}}{c} \gtrsim c^{\frac{8}{3}}, \quad c^{\frac{17}{12}} \lesssim\left\|w_{x}^{+}(t)\right\|_{L^{2}}+c^{\frac{1}{2}}\left\|w^{+}(t)\right\|_{L^{2}} \lesssim c^{\frac{11}{12}} .
$$

The first part of the theorem means that the two solitons are preserved through the collision, even the smaller one. Indeed, for $c$ small, $\sup _{t}\left\|w^{+}(t)\right\|_{H^{1}} \lesssim c^{\frac{1}{3}} \ll\left\|Q_{c}\right\|_{H^{1}} \sim c^{\frac{1}{12}}$.

The second part of the theorem says that the sizes of the final solitons as $t \rightarrow+\infty$ are slightly changed with respect to their original sizes as $t \rightarrow-\infty$, and that the residue does not converge to zero. In particular, the solution is not a pure 2 -soliton as $t \rightarrow+\infty$ in this regime. Thus, the collision is not elastic.

7.2. Collision for the quartic gKdV equation II. A first intuition on the general problem of two solitons with almost same sizes comes from the explicit multi-solitons of the integrable case. From [93], we have a sharp description of the behavior of the multi-soliton of (1) satisfying

$$
\lim _{t \rightarrow \pm \infty}\left\|u(t)-Q_{c_{1}}\left(\cdot-c_{1} t-\sigma_{1}^{ \pm}\right)-Q_{c_{2}}\left(\cdot-c_{2} t-\sigma_{2}^{ \pm}\right)\right\|_{H^{1}}=0
$$

in the special asymptotics where $0<\mu_{0}=\frac{c_{2}-c_{1}}{c_{1}+c_{2}} \ll 1$, i.e. for nearly equal solitons. Indeed, the following global in time estimate is proved for some explicit functions $c_{k}(t), \sigma_{k}(t)$ :

$$
\sup _{t, x \in \mathbb{R}}\left|u(t, x)-Q_{c_{1}(t)}\left(x-\sigma_{1}(t)\right)-Q_{c_{2}(t)}\left(x-\sigma_{2}(t)\right)\right| \lesssim \mu_{0}^{2} .
$$

Moreover, it is proved that $\min _{t \in \mathbb{R}}\left(\sigma_{1}(t)-\sigma_{2}(t)\right) \sim 2\left|\ln \mu_{0}\right|$. This means that the minimum separation between the two solitons is large when $\mu_{0} \ll 1$. In particular, the two solitons never cross and the solution has two maximum points for all time. The interaction is repulsive, the solitons exchange their sizes and speeds at large distance and consequently avoid the collision.

We now recall results from [116] for the quartic gKdV equation. Let $u$ be a solution of (8) for which the initial data is close to the sum $Q(x)+Q_{c}\left(x+Y_{0}\right)$, where $Y_{0}>0$ is large and $0 \leq c-1 \leq \exp \left(-\frac{1}{2} Y_{0}\right)$, so that the quicker soliton can be initially on the left of the other soliton. It follows from [116] that the interaction of the two solitons is repulsive: the two solitons remain separated for all positive time and eventually $u(t)$ behaves as

$$
u(t, x)=Q_{c_{1}^{+}}\left(x-c_{1}^{+} t-\sigma_{1}^{+}\right)+Q_{c_{2}^{+}}\left(x-c_{2}^{+} t-\sigma_{2}^{+}\right)+w(t, x),
$$


for large time, for some $c_{1}^{+}>c_{2}^{+}$close to 1 and $w$ small in some sense. The situation for almost equal solitons of the quartic $(\mathrm{gKdV})$ is thus at the main order similar to the one described in the integrable case in [93]. The analysis part in [116] relies on techniques from [60,61] and on the use of spaces introduced in this context in [125].

Before presenting the main result from [103], for simplicity, we change variables. For $c_{2}-c_{1}>0$ small, and any $\sigma_{1}, \sigma_{2}$, let $u(t)$ be the unique solution of (8) such that

$$
\lim _{t \rightarrow-\infty}\left\|u(t)-Q_{c_{1}}\left(\cdot-c_{1} t-\sigma_{1}\right)-Q_{c_{2}}\left(\cdot-c_{2} t-\sigma_{2}\right)\right\|_{H^{1}}=0 .
$$

Let

$$
c_{0}=\frac{c_{1}+c_{2}}{2}, \quad \mu_{0}=\frac{c_{2}-c_{1}}{c_{1}+c_{2}}, \quad y_{1}=\sigma_{1} \sqrt{c_{0}}, \quad y_{2}=\sigma_{2} \sqrt{c_{0}} .
$$

Then the function $U(t, x)=c_{0}^{-1 / 3} u\left(c_{0}^{-3 / 2} t, c_{0}^{-1 / 2}(x+t)\right)$ solves

$$
\partial_{t} U+\partial_{x}\left(\partial_{x}^{2} U-U+U^{4}\right)=0, \quad(t, x) \in \mathbb{R} \times \mathbb{R},
$$

and is the unique solution of (9) satisfying

$$
\lim _{t \rightarrow-\infty}\left\|U(t)-Q_{1-\mu_{0}}\left(\cdot+\mu_{0} t-y_{1}\right)-Q_{1+\mu_{0}}\left(\cdot-\mu_{0} t-y_{2}\right)\right\|_{H^{1}}=0 .
$$

The next result concerns the asymptotics $\mu_{0}>0$ small.

Theorem 13 (Inelastic interaction of two nearly equal solitons, [103]). Assume $0<\mu_{0} \ll 1$. Let $U$ be the unique solution of (9) such that

$$
\lim _{t \rightarrow-\infty}\left\|U(t)-Q_{1-\mu_{0}}\left(\cdot+\mu_{0} t+Y_{0}+\ln 2\right)-Q_{1+\mu_{0}}\left(\cdot-\mu_{0} t-Y_{0}-\ln 2\right)\right\|_{H^{1}}=0,
$$

where $Y_{0}=\frac{1}{2}\left|\ln \left(\mu_{0}^{2} / \alpha\right)\right|$ and $\alpha=12(10)^{2 / 3}\left(\int Q^{2}\right)^{-1}$. Then the following holds.

(i) Global stability of the 2 -soliton behavior. There exist $\mu_{1}, \mu_{2}, y_{1}, y_{2}$ such that

$$
\begin{aligned}
& w(t, x)=U(t)-Q_{1+\mu_{1}(t)}\left(x-y_{1}(t)\right)-Q_{1+\mu_{2}(t)}\left(x-y_{2}(t)\right) \\
& \text { satisfies }\left|\min _{t \in \mathbb{R}}\left(y_{1}(t)-y_{2}(t)\right)-2 Y_{0}\right| \lesssim \mu_{0} \text { and } \\
& \lim _{t \rightarrow+\infty}\|w(t)\|_{H^{1}\left(x>-\frac{9}{10} t\right)}=0, \quad \sup _{t \in \mathbb{R}} \mid w(t) \|_{H^{1}} \lesssim \mu_{0}^{\frac{3}{2}} .
\end{aligned}
$$

(ii) Inelasticity of the interaction.

$$
\lim _{+\infty} \mu_{1}-\mu_{0} \gtrsim \mu_{0}^{5}, \quad \mu_{0}-\lim _{+\infty} \mu_{2} \gtrsim \mu_{0}^{5}, \quad \liminf _{t \rightarrow+\infty}\|w(t)\|_{H^{1}} \gtrsim \mu_{0}^{3} .
$$

It follows that no pure 2-soliton exists also in this regime. The proofs of Theorems 12 and 13 are based on the construction of a refined approximate solution of the two-soliton problem for all $t$ and $x$.

7.3. Collision for the quartic gKdV equation III. Still concerning the collision of two solitons for the quartic gKdV equation, we recall from [104] the following negative result.

Theorem 14 (Inelasticity of collision for gKdV, [104]). Let $K \geq 2,0<c_{K}<\cdots<c_{1}=1$ and $\sigma_{1}, \ldots, \sigma_{K} \in \mathbb{R}$. Let $u$ be the solution of (8) satisfying

$$
\lim _{t \rightarrow-\infty}\left\|u(t)-\sum_{k=1}^{K} Q_{c_{k}}\left(\cdot-c_{k} t-\sigma_{k}\right)\right\|_{H^{1}}=0 .
$$

Assume that $\sum_{k=2}^{K}\left(1-c_{k}\right)^{2}<\frac{1}{16}$. Then, $u(t)$ is not an asymptotic multi-soliton as $t \rightarrow+\infty$. In particular, there exists no pure multi-soliton of (1) with the speeds $c_{1}, c_{2}, \ldots, c_{K}$. 
In the case of two solitons, the condition on the speeds reduces to $\frac{3}{4} c_{1}<c_{2}<c_{1}$. In contrast with Theorems 12 and 13, the result in Theorem 14 is not perturbative and the explicit condition on the speeds seems technical. The strategy of the proof of Theorem 14 is to study the asymptotic behavior of $u(t, x)$ for any $t$ and for any large $x$ (i.e. far from the collision region, which seems impossible to describe in the general case) and to find a contradiction with the fact that $u$ is an asymptotic two-soliton in the two directions of time. Being a proof by contradiction, it does not give further information on the collision.

7.4. Collision for the perturbed integrable NLS equation. Let $\beta>0$ and $0<c \ll 1$. Under the following assumptions for the perturbation $|f(u)| \lesssim_{0} u^{2}, f(u) \lesssim|u|^{q}(q<2)$, it is proved in [127], that there exists a solution $u$ of

$$
i \partial_{t} u+\partial_{x}^{2} u+|u|^{2} u+f\left(|u|^{2}\right) u=0, \quad(t, x) \in \mathbb{R} \times \mathbb{R},
$$

satisfying $\lim _{t \rightarrow-\infty}\left\|u(t)-e^{i t} Q-e^{i \Gamma_{\beta}(t, \cdot)} Q_{c}(\cdot-\beta t)\right\|_{H^{1}} \rightarrow 0$, and for which the small soliton splits in two after the collision, in the following sense

$$
u(t, x) \sim e^{i \Gamma(t, x)} Q(x-\sigma(t))+\psi^{+}(t, x)+\psi^{-}(t, x),
$$

where $\psi^{ \pm}$are solutions of (2) corresponding to the transmitted part and the reflected part of the small soliton. The above estimate holds on large time intervals after the collision depending on $1 / c$. The splitting means some strong form of inelasticity.

7.5. Collision for the $5 \mathrm{D}$ energy critical wave equation. In view of the results described so far, it was natural to search a situation where a non-perturbative approach would allow to treat all two-soliton collisions. In the case of the 5D energy critical wave equation, we now recall from [106] a result showing the inelastic nature of the collision of any two solitons, except the special case of same scaling and opposite signs.

Theorem 15 (Inelasticity of soliton collisions for NLW, [106]). Let $d=5$. For $k \in\{1,2\}$, let $\lambda_{k}^{\infty}>0, \mathbf{y}_{k}^{\infty} \in \mathbb{R}^{5}, \epsilon_{k} \in\{ \pm 1\}, \ell_{k} \in \mathbb{R}^{5}$ with $\left|\ell_{k}\right|<1$, and

$$
W_{k}^{\infty}(t, x)=\frac{\epsilon_{k}}{\left(\lambda_{k}^{\infty}\right)^{\frac{3}{2}}} W_{\ell_{k}}\left(\frac{x-\boldsymbol{\ell}_{k} t-\mathbf{y}_{k}^{\infty}}{\lambda_{k}^{\infty}}\right) .
$$

Assume that $\ell_{1} \neq \ell_{2}$ and $\epsilon_{1} \lambda_{1}^{\infty}+\epsilon_{2} \lambda_{2}^{\infty} \neq 0$. Then, there exists a solution $u$ of (6) in the energy space such that

(i) Two-soliton as $t \rightarrow-\infty$

$$
\lim _{t \rightarrow-\infty}\left\|\nabla_{t, x} u(t)-\nabla_{t, x}\left(W_{1}^{\infty}(t)+W_{2}^{\infty}(t)\right)\right\|_{L^{2}}=0 .
$$

(ii) Dispersion as $t \rightarrow+\infty$. For all $A>0$ large enough,

$$
\liminf _{t \rightarrow+\infty}\|\nabla u(t)\|_{L^{2}(|x|>t+A)} \gtrsim A^{-\frac{5}{2}} .
$$

Note first that the solution constructed in Theorem 15 is a two-soliton asymptotically as $t \rightarrow-\infty$ and that it does not necessarily exist for all $t \in \mathbb{R}$. However, by finite speed of propagation and small data Cauchy theory, it is straightforward to justify that it can be extended uniquely as a solution of (6) for all $t \in \mathbb{R}$ in the region $|x|>|t|+A$, provided that $A$ is large enough. Thus, the limit in (10) makes sense. Since the estimate (10) gives an explicit lower bound on the loss of energy as dispersion as $t \rightarrow+\infty$, the solution $u$ is not a two-soliton asymptotically as $t \rightarrow+\infty$ and the collision is inelastic. Note that the two-soliton 
could have any global behavior, like dislocation of the solitons and dispersion, blow-up or a different multi-soliton plus radiation, but the property obtained is universal.

The only case left open by Theorem 15 corresponds to the dipole case. It is the first result proving inelasticity rigorously without restriction on the relative sizes or speeds of the solitons except an exceptional case.

The strategy of the proof is to construct a refined approximate solution of the two-soliton problem for large negative times that displays an explicit dispersive radial part at the leading order and then to propagate the dispersion for any positive time at the exterior of large cones by finite speed of propagation and the method of channels of energy from [43, 75]. To finish, we recall such a typical result of channel of energy for the radial linear wave equation in 5D from [75], Proposition 4.1 (see also [42, 43, 74]).

Theorem 16 (Exterior energy estimates for the 5D linear wave equation, [75]). Any radial energy solution $U_{\mathrm{L}}$ of the $5 \mathrm{D}$ linear wave equation

$$
\left\{\begin{array}{l}
\partial_{t}^{2} U_{\mathrm{L}}-\Delta U_{\mathrm{L}}=0, \quad(t, x) \in \mathbb{R} \times \mathbb{R}^{5}, \\
U_{\mathrm{L} \mid t=0}=U_{0} \in \dot{H}^{1}, \quad \partial_{t} U_{\mathrm{L} \mid t=0}=U_{1} \in L^{2},
\end{array}\right.
$$

satisfies, for any $R>0$,

$$
\sum_{ \pm}\left\{\limsup _{t \rightarrow \pm \infty} \int_{|x|>|t|+R}\left(\left|\partial_{t} U_{\mathrm{L}}(t, x)\right|^{2}+\left|\nabla U_{\mathrm{L}}(t, x)\right|^{2}\right) d x\right\} \gtrsim\left\|\pi_{R}^{\perp}\left(U_{0}, U_{1}\right)\right\|_{\left(\dot{H}^{1} \times L^{2}\right)(|x|>R)}^{2}
$$

where $\pi_{R}^{\perp}\left(U_{0}, U_{1}\right)$ denotes the orthogonal projection of $\left(U_{0}, U_{1}\right)$ onto the complement of the plane $\operatorname{span}\left\{\left(|x|^{-3}, 0\right),\left(0,|x|^{-3}\right)\right\}$ in $\left(\dot{H}^{1} \times L^{2}\right)(|x|>R)$.

\section{REFERENCES}

[1] M. J. Ablowitz, D. Klaup, A. Newell and H. Segur, The inverse scattering transform-Fourier analysis for nonlinear problems. Studies in Appl. Math. 53 (1974), no. 4, 249-315.

[2] M. J. Ablowitz, M. D. Kruskal and J. F. Ladik, Solitary wave collisions. SIAM J. Appl. Math. 36 (1979), 428-437.

[3] M. Alejo and C. Muñoz, Nonlinear stability of mKdV breathers. Comm. Math. Phys. 324 (2013), no. 1, 233-262.

[4] M. Beceanu, A centre-stable manifold for the focussing cubic NLS in $\mathbb{R}^{1+3}$. Comm. Math. Phys. 280 (2008), no.1, 145-205.

[5] M. Beceanu, A critical center-stable manifold for Schrödinger's equation in three dimensions. Comm. Pure Appl. Math. 65 (2012), no. 4, 431-507.

[6] T. B. Benjamin, The stability of solitary waves. Proc. Roy. Soc. London A 328, (1972) 153-183.

[7] H. Berestycki and P.-L. Lions, Nonlinear scalar field equations. I. Existence of a ground state. Arch. Rational Mech. Anal. 82: 313-345, 1983.

[8] J. L. Bona, On the stability theory of solitary waves. Proc. Roy. Soc. London A 349, (1975) 363-374.

[9] J. L. Bona, W. G. Pritchard and L. R. Scott, Solitary-wave interaction. Phys. Fluids 23, 438 (1980).

[10] J. L. Bona, P. E. Souganidis, and W. A. Strauss, Stability and instability of solitary waves of Korteweg-de Vries type. Proc. Roy. Soc. London Ser. A 411 (1987), no. 1841, 395-412.

[11] M. Borghese, R. Jenkins, K. T.-R. McLaughlin, Long-time asymptotic behavior of the focusing nonlinear Schrödinger equation. Preprint arXiv:1604.07436

[12] V. Buslaev and G. Perelman, Scattering for the nonlinear Schrödinger equations: states close to a soliton. St. Petersburgh Math. J. 4 (1993), no. 6, 1111-1142.

[13] V. Buslaev and G. Perelman, On the stability of solitary waves for nonlinear Schrödinger equations. Nonlinear evolution equations, 75-98, Amer. Math. Soc. Transl. Ser. 2, 164, AMS, Providence, RI, 1995.

[14] V. Buslaev and C. Sulem, On asymptotic stability of solitary waves for nonlinear Schrödinger equations. Ann. Inst. H. Poincaré Anal. Non Linéaire, 20 (2003), no. 3, 419-475. 
[15] T. Cazenave, Semilinear Schrödinger equations. New York University, Courant Institute, New York, 2003.

[16] T. Cazenave and P.L. Lions, Orbital stability of standing waves for some nonlinear Schrödinger equations. Comm. Math. Phys. 85, (1982) 549-561.

[17] A. Cohen, Existence and regularity for solutions of the Korteweg-de Vries equation. Arch. Rat. Mech. Anal. 71 (1979), 143-175.

[18] V. Combet, Multi-soliton solutions for the supercritical gKdV equations. Comm. Partial Differential Equations 36 (2010), no. 3, 380-419.

[19] V. Combet, Multi-existence of multi-solitons for the super-critical nonlinear Schrödinger equation in one dimension. Discrete Contin. Dyn. Syst. 34 (2014), no. 5, 1961-1993.

[20] V. Combet and Y. Martel, Sharp asymptotics for the minimal mass blow up solution of the critical gKdV equation. Bull. Sci. Math. 141 (2017), no. 2, 20-103.

[21] V. Combet and Y. Martel, Construction of multi-bubble solutions for the critical gKdV equation. Preprint arXiv:1706.09870

[22] O. Costin, M. Huang and W. Schlag, On the spectral properties of $L_{ \pm}$in three dimensions. Nonlinearity 25 (2012), 125-164.

[23] R. Côte, On the soliton resolution for equivariant wave maps to the sphere. Comm. Pure Appl. Math. 68 (2015), no. 11, 1946-2004.

[24] R. Côte, C. E. Kenig, A. Lawrie and W. Schlag, Characterization of large energy solutions of the equivariant wave map problem: I. Amer. J. Math. 137 (2015), no. 1, 139-207.

[25] R. Côte, C. E. Kenig, A. Lawrie and W. Schlag, Characterization of large energy solutions of the equivariant wave map problem: II. Amer. J. Math. 137 (2015), no. 1, 209-250.

[26] R. Côte and Y. Martel, Multi-travelling waves for the nonlinear Klein-Gordon equation. To appear in Trans. Amer. Math. Soc. arXiv:1612.02625

[27] R. Côte, Y. Martel and F. Merle, Construction of multi-soliton solutions for the $L^{2}$-supercritical gKdV and NLS equations. Rev. Mat. Iberoamericana 27 (2011), 273-302.

[28] R. Côte and C. Muñoz, Multi-solitons for nonlinear Klein-Gordon equations. Forum of Mathematics, Sigma 2 (2014)

[29] W. Craig, P. Guyenne, J. Hammack, D. Henderson and C. Sulem, Solitary water wave interactions. Phys. Fluids 18, 057106 (2006).

[30] S. Cuccagna, On asymptotic stability in 3D of kinks for the $\phi^{4}$ model. Trans. Amer. Math. Soc. 360 (2008), no. 5, 2581-2614.

[31] S. Cuccagna, On asymptotic stability of moving ground states of the nonlinear Schrödinger equation. Trans. Amer. Math. Soc. 366 (2014), no. 2827-2888.

[32] S. Cuccagna and D. Pelinovsky, The asymptotic stability of solitons in the cubic NLS equation on the line. Applicable Analysis 93 (2014), no. 4, 791-822.

[33] S. Cuenda, N. R. Quintero and A. Sánchez, Sine-Gordon wobbles through Bäcklund transformations. Discrete and Continuous Dynamical Systems - Series S 4 (2011), 1047-1056.

[34] T. Dauxois and M. Peyrard, Physics of solitons. Cambridge University Press, Cambridge, 2010.

[35] P. Deift and E. Trubowitz, Inverse scattering on the line. Comm. Pure Appl. Math. 32 (1979), no. 2, $121-251$

[36] P. Deift, S. Venakides and X. Zhou, The collisionless shock region for the long-time behavior of solutions of the KdV equation. Comm. Pure Appl. Math. 47 (1994), no. 2, 199-206.

[37] P. A. Deift and X. Zhou, Long-time asymptotics for integrable nonlinear wave equations. Important developments in soliton theory, 181-204, Springer Ser. Nonlinear Dynam., Springer, Berlin, 1993.

[38] P. A. Deift and X. Zhou, Perturbation theory for infinite-dimensional integrable systems on the line. A case study. Acta Math. 188 (2002), no. 2, 163-262.

[39] R. K. Dodd, J. C. Eilbeck, J. D. Gibbona and H. C. Morris, Solitons and nonlinear wave equations. Academic Press, Inc., London-New York, 1982.

[40] T. Duyckaerts and F. Merle, Dynamics of threshold solutions for energy-critical wave equation. Int. Math. Res. Pap. 2008, Art ID rpn002.

[41] T. Duyckaerts, C. E. Kenig and F. Merle, Universality of blow-up profile for small radial type II blow-up solutions of the energy-critical wave equation. J. Eur. Math. Soc. 13 (2011), 533-599.

[42] T. Duyckaerts, C. E. Kenig and F. Merle, Universality of the blow-up profile for small type II blow-up solutions of the energy-critical wave equation : the non-radial case. J. Eur. Math. Soc. 14 (2012), no. 5, $1389-1454$. 
[43] T. Duyckaerts, C. E. Kenig and F. Merle, Classification of radial solutions of the focusing, energy-critical wave equation. Cambridge Journal of Mathematics 1 (2013), 75-144.

[44] T. Duyckaerts, C. E. Kenig and F. Merle, Solutions of the focusing nonradial critical wave equation with the compactness property. Ann. Sc. Norm. Super. Pisa Cl. Sci. (5) 15 (2016), 731-808.

[45] T. Duyckaerts, H. Jia, C. E. Kenig and F. Merle, Soliton resolution along a sequence of times for the focusing energy critical wave equation. Geom. Funct. Anal. 27 (2017), no. 4, 798-862.

[46] W. Eckhaus and P. Schuur. The emergence of solutions of the Korteweg-de Vries equation from arbitrary initial conditions. Math. Meth. Appl. Sci. 5, (1983) 97-116.

[47] S.-I. Ei and T. Ohta, The motion of interacting pulses. Phys. Rev. 50 (1994), 4672-4678.

[48] L. D. Faddeev and L. A. Takhtajan, Hamiltonian Methods in the Theory of Solitons. Translated from the 1986 Russian original by Alexey G. Reyman. Reprint of the 1987 English edition. Classics in Mathematics. Springer, Berlin, 2007.

[49] E. Fermi, J. Pasta and S. Ulam, Studies of nonlinear problems, I, Los Alamos Report LA1940 (1955); reproduced in Nonlinear Wave Motion, A. C. Newell, ed., AMS, Providence, R.I., 1974, pp. 143-156.

[50] C. S. Gardner, C. S. Greene, M. D. Kruskal and R. M. Miura, Method for solving the Korteweg-de Vries equation, Phys. Rev. Lett. 19 (1967), 1095-1087.

[51] C. S. Gardner, C. S. Greene, M. D. Kruskal and R. M. Miura, Korteweg-de Vries equation and generalization. VI. Methods for exact solution. Comm. Pure Appl. Math., 27: 97-133, 1974.

[52] P. Gérard, E. Lenzmann, O. Pocovnicu and P. Raphaël, A two-soliton with transient turbulent regime for the cubic half-wave equation on the real line. Preprint arXiv:1611.08482

[53] S. Gustafson, K. Nakanishi, and T.-P. Tsai, Asymptotic stability and completeness in the energy space for nonlinear Schrödinger equations with small solitary waves. Int. Math. Res. Not. (2004), no. 66, 3559-3584.

[54] P. Germain, F. Pusateri and F. Rousset, Asymptotic stability of solitons for mKdV. Adv. Math. 299 (2016), 272-330.

[55] J. Ginibre and G. Velo, On a class of nonlinear Schrödinger equations. I. The Cauchy problem, general case. J. Funct. Anal. 32, (1979) 1-32.

[56] K. A. Gorshkov and L. A. Ostrovsky, Interactions of solitons in nonintegrable systems: direct perturbation method and applications. Physica 3D (1981), 428-438.

[57] M. Grillakis, Analysis of the linearization around a critical point of an infinite dimensional hamiltonian system. Comm. Pure Appl. Math. 41 (1988), 747-774.

[58] M. Grillakis, J. Shatah and W. Strauss, Stability theory of solitary waves in the presence of symmetry. I. J. Func. Anal. 74 (1987), 160-197.

[59] J. Hammack, D. Henderson, P. Guyenne and Ming Yi, Solitary-wave collisions, in Proceedings of the 23rd ASME Offshore Mechanics and Artic Engineering (A symposium to honor Theodore Yao-Tsu Wu), Vancouver, Canada, June 2004 (Word Scientific, Singapore, 2004).

[60] N. Hayashi and P. Naumkin, Large time asymptotics of solutions to the generalized Korteweg-de Vries equation. J. Funct. Anal. 159 (1998), 110-136.

[61] N. Hayashi and P. Naumkin, On the modified Korteweg-de Vries equation. Math. Phys. Anal. Geom. 4 (2001), 197-201.

[62] D. B. Henry, J. F. Perez, and W. F. Wreszinski, Stability theory for solitary-wave solutions of scalar field equations. Comm. Math. Phys. 85 (1982), no. 3, 351-361.

[63] R. Hirota, Exact solution of the Korteweg-de Vries equation for multiple collisions of solitons. Phys. Rev. Lett. 27 (1971), 1192-1194.

[64] J. Holmer and M. Zworski, Slow soliton interaction with delta impurities. J. Mod. Dyn. 1 (2007), no. 4, 689-718

[65] J. Holmer and M. Zworski, Soliton interaction with slowly varying potentials. Int. Math. Res. Not. 2008, no. 10, Art. ID rnn026.

[66] J. Holmer, J. Marzuola and M. Zworski, Soliton splitting by external delta potentials. J. Nonlinear Sci. 17 (2007), no. 4, 349-367.

[67] J. Holmer, J. Marzuola and M. Zworski, Fast soliton scattering by delta impurities. Comm. Math. Phys. 274 (2007), no. 1, 187-216.

[68] J. Jendrej, Construction of type II blow-up solutions for the energy-critical wave equation in dimension 5. J. Funct. Anal. 272 (2017), no. 3, 866-917.

[69] J. Jendrej, Construction of two-bubble solutions for energy-critical wave equations. To appear in Amer. J. Math. Preprint arXiv:1602.06524 
[70] J. Jendrej and A. Lawrie, Two-bubble dynamics for threshold solutions to the wave maps equation. Preprint arXiv:1706.00089

[71] R. Jenkins, J. Liu, P. Perry and C. Sulem, Soliton resolution for the derivative non-linear Schrödinger equation. Preprint arXiv:1710.03819

[72] V. I. Karpman and V. V. Solov'ev, A perturbational approach to the two-soliton systems. Physica 3D (1981), 487-502.

[73] T. Kato, On the Cauchy problem for the (generalized) Korteweg-de Vries equation. Advances in Mathematical Supplementary Studies, Studies in Applied Math. 8 (1983), 93-128.

[74] C. E. Kenig, A. Lawrie, B. Liu and W. Schlag, Channels of energy for the linear radial wave equation. Adv. Math. 285 (2015), 877-936.

[75] C. E. Kenig, A. Lawrie and W. Schlag, Relaxation of wave maps exterior to a ball to harmonic maps for all data. Geom. Funct. Anal. 24 (2014), 610-647.

[76] C.E. Kenig, Y. Martel, Asymptotic stability of solitons for the Benjamin-Ono equation. Revista Matematica Iberoamericana 25 (2009), 909-970.

[77] C. E. Kenig and F. Merle, Global well-posedness, scattering and blow-up for the energy-critical focusing non-linear wave equation. Acta Math. 201 (2008), 147-212.

[78] C. E. Kenig, G. Ponce and L. Vega, Well-posedness and scattering results for the generalized Korteweg-de Vries equation via the contraction principle. Comm. Pure Appl. Math. 46 (1993), 527-620.

[79] Y. S. Kivshar and B. Malomed, Dynamics of solitons in nearly integrable models. Review of Modern Physics, 61 (1989), 763-915.

[80] E. Kopylova and A. I. Komech, On asymptotic stability of kink for relativistic Ginzburg-Landau equations. Arch. Ration. Mech. Anal. 202 (2011), no. 1, 213-245.

[81] E. Kopylova and A. I. Komech, On asymptotic stability of moving kink for relativistic Ginzburg-Landau equation. Comm. Math. Phys. 302 (2011), no. 1, 225-252.

[82] M. Kowalczyk, Y. Martel and C. Muñoz, Kink dynamics in the $\phi^{4}$ model: asymptotic stability for odd perturbations in the energy space. J. Amer. Math. Soc. 30 (2017), 769-798.

[83] M. Kowalczyk, Y. Martel and C. Muñoz, On asymptotic stability of nonlinear waves. Séminaire Laurent Schwartz - EDP et applications (2016-2017), Exp. No. 18, 27 p.

[84] J. Krieger, Y. Martel and P. Raphaël, Two-soliton solutions to the three-dimensional gravitational Hartree equation. Comm. Pure Appl. Math. 62 (2009), no. 11, 1501-1550.

[85] J. Krieger, K. Nakanishi and W. Schlag, Threshold phenomenon for the quintic wave equation in three dimensions. Comm. Math. Phys. 327 (2014), no. 1, 309-332.

[86] J. Krieger, K. Nakanishi and W. Schlag, Global dynamics away from the ground state for the energycritical nonlinear wave equation. Amer. J. Math. 135 (2013), no. 4, 935-965.

[87] J. Krieger, W. Schlag and D. Tataru, Slow blow-up solutions for the $H^{1}\left(\mathbb{R}^{3}\right)$ critical focusing semilinear wave equation. Duke Math. J. 147 (2009), no. 1, 1-53.

[88] M. D. Kruskal, The Korteweg-de Vries equation and related evolution equations, in Nonlinear Wave Motion, A.C. Newell, ed., American Mathematical Society, Providence, R. I., 1974, pp. 61-83.

[89] S. B. Kuksin, Analysis of Hamiltonian PDEs, Oxford Lecture Series in Mathematics and its Applications, 19. Oxford University Press, Oxford, 2000.

[90] M. K. Kwong. Uniqueness of positive solutions of $\Delta u-u+u^{p}=0$ in $\mathbb{R}^{n}$. Arch. Rational Mech. Anal., 105(3):243-266, 1989.

[91] G. L. Lamb Jr., Element of soliton theory (John Wiley \& Sons, New York 1980).

[92] P. D. Lax, Integrals of nonlinear equations of evolution and solitary waves. Comm. Pure Appl. Math. 21 (1968), 467-490.

[93] R. LeVeque, On the interaction of nearly equal solitons in the KdV equations. SIAM J. Appl. Math. 47 (1987), 254-262.

[94] Y. Li and D. H. Sattinger, Soliton collisions in the ion acoustic plasma equations. J. Math. Fluid Mech. 1 (1999), 117-130.

[95] N. Manton and P. Sutcliffe, Topological solitons, Cambridge Monographs on Mathematical Physics, Cambridge University Press, Cambridge, 2004.

[96] Y. Martel, Asymptotic $N$-soliton-like solutions of the subcritical and critical generalized Korteweg-de Vries equations. Amer. J. Math. 127 (2005), 1103-1140.

[97] Y. Martel and F. Merle, A Liouville Theorem for the critical generalized Korteweg-de Vries equation. $J$. Math. Pures Appl. 79, (2000) 339-425. 
[98] Y. Martel and F. Merle, Asymptotic stability of solitons for subcritical generalized KdV equations. Arch. Ration. Mech. Anal. 157, (2001) 219-254.

[99] Y. Martel and F. Merle, Stability of blow up profile and lower bounds for blow up rate for the critical generalized KdV equation. Ann. of Math. 155, (2002) 235-280.

[100] Y. Martel and F. Merle, Asymptotic stability of solitons for subcritical gKdV equations revisited. Nonlinearity 18 (2005), no. 1, 55-80.

[101] Y. Martel and F. Merle, Multi-solitary waves for nonlinear Schrödinger equations. Annales de l'IHP (C) Non Linear Analysis 23 (2006), 849-864.

[102] Y. Martel and F. Merle, Description of two soliton collision for the quartic gKdV equation. Ann. Math. (2) $\mathbf{1 7 4}$, no. 2 (2011), 757-857.

[103] Y. Martel and F. Merle, Inelastic interaction of nearly equal solitons for the quartic gKdV equation. Invent. Math. 183, no. 3, 563-648 (2011).

[104] Y. Martel and F. Merle, On the nonexistence of pure multi-solitons for the quartic gKdV equation. Internat. Math. Res. Notices (2015) (3): 688-739.

[105] Y. Martel and F. Merle, Construction of multi-solitons for the energy-critical wave equation in dimension 5. Arch. Ration. Mech. Anal. 222 (2016), no. 3, 1113-1160.

[106] Y. Martel and F. Merle, Inelasticity of soliton collisions for the 5D energy critical wave equation. Preprint arXiv:1708.09712

[107] Y. Martel, F. Merle and P. Raphaël, Blow up for the critical generalized Korteweg de Vries equation. I: Dynamics near the soliton. Acta Math. 212 (2014), no. 1, 59-140.

[108] Y. Martel, F. Merle and T.-P. Tsai, Stability and asymptotic stability in the energy space of the sum of $N$ solitons for subcritical gKdV equations. Commun. Math. Phys. 231, (2002) 347-373.

[109] Y. Martel and P. Raphaël, Strongly interacting blow up bubbles for the mass critical NLS. To appear in Ann. Sci. Éc. Norm. Supér. Preprint arxiv 1512.00900

[110] F. Merle, Construction of solutions with exactly $k$ blow-up points for the Schrödinger equation with critical nonlinearity. Comm. Math. Phys. 129 (1990), no. 2, 223-240.

[111] F. Merle and P. Raphaël, On universality of blow-up profile for $L^{2}$ critical nonlinear Schrödinger equation. Invent. Math., 156(3):565-672, 2004.

[112] F. Merle and P. Raphaël, Profiles and quantization of the blow up mass for critical nonlinear Schrödinger equation. Comm. Math. Phys. 253 (2005), no. 3, 675-704.

[113] M. Ming, F. Rousset and N. Tzvetkov, Multi-solitons and related solutions for the water-waves system. SIAM J. Math. Anal. 47 (2015), no. 1, 897-954.

[114] R. M. Miura, The Korteweg-de Vries equation, a survey of results. SIAM Review 18 (1976), 412-459.

[115] R. M. Miura, C. S. Gardner and M. D. Kruskal, Korteweg-deVries equation and generalizations. Existence of conservation laws and constants of motion. J. Math. Phys. 9 (1968), 1204-1209.

[116] T. Mizumachi, Weak interaction between solitary waves of the generalized KdV equations. SIAM J. Math. Anal. 35 (2003), no. 4, 1042-1080.

[117] C. Muñoz, On the inelastic two-soliton collision for gKdV equations with general nonlinearity. Int. Math. Res. Not. 2010, no. 9, 1624-1719.

[118] C. Muñoz, Inelastic character of solitons of slowly varying gKdV equations. Comm. Math. Phys. 314 (2012), no. 3, 817852 .

[119] C. Muñoz, Dynamics of soliton-like solutions for slowly varying, generalized KdV equations: refraction versus reflection. SIAM J. Math. Anal. 44 (2012), no. 1, 1-60.

[120] K. Nakanishi and W. Schlag, Invariant manifolds and dispersive Hamiltonian evolution equations. Zurich Lectures in Advanced Mathematics. European Mathematical Society (EMS), Zïich, 2011.

[121] V. J. Novoksenov, Asymptotic behavior as $t \rightarrow \infty$ of the solution to the Cauchy problem for a nonlinear Schrödinger equation. Dokl. Akad. Nauk SSSR 251 (1980), 799-802.

[122] T. V. Nguyen, Existence of multi-solitary waves with logarithmic relative disctances for the NLS equation. Preprint arXiv:1611.08869

[123] T. V. Nguyen, Strongly interacting multi-solitons with logarithmic relative distance for gKdV equation. To appear in Nonlinearity.

[124] E. Olmedilla, Multiple pole solutions of the nonlinear Schrödinger equation. Physica D 25 (1987), 330346.

[125] R. L. Pego and M I. Weinstein, Eigenvalues, and instabilities of solitary waves. Philos. Trans. Roy. Soc. London Ser. A 340 (1992), no. 1656, 47-94. 
[126] R. L. Pego and M I. Weinstein, Asymptotic stability of solitary waves. Comm. Math. Phys. 164, (1994) 305-349.

[127] G. Perelman, Two soliton collision for nonlinear Schrödinger equations in dimension 1. Ann. Inst. H. Poincaré Anal. Non Linéaire 28 (2011), no. 3, 357-384.

[128] P. Raphaël, On singularity formation in Hamiltonian evolution equations. Proceedings of the International Congress of Mathematicians, Seoul, 2014

[129] I. Rodnianski, W. Schlag, and A. Soffer, Dispersive analysis of charge transfer models. Comm. Pure Appl. Math. 58 (2005), no. 2, 149-216.

[130] I. Rodnianski, W. Schlag, and A. Soffer, Asymptotic stability of $N$-soliton states of NLS. Preprint arXiv:math/0309114

[131] W. Schlag, Spectral theory and nonlinear partial differential equations: a survey. Discrete Contin. Dyn. Syst. 15 (2006), no. 3, 703-723.

[132] W. Schlag, Dispersive estimates for Schrödinger operators: A survey. Mathematical aspects of nonlinear dispersive equations, 255-285, Ann. of Math. Stud., 163, Princeton Univ. Press, Princeton, NJ, 2007.

[133] W. Schlag, Stable manifolds for an orbitally unstable nonlinear Schrödinger equation. Ann. of Math. (2) 169 (2009), no. 1, 139-227.

[134] P. C. Schuur, Asymptotic analysis of solitons problems. Lecture Notes in Math. 1232 (1986), SpringerVerlag, Berlin.

[135] H. Segur, Wobbling kinks in $\varphi^{4}$ and sine-Gordon theory. J. Math. Phys. 24 (1983), no. 6, 1439-1443.

[136] L. Y. Shih, Soliton-like interaction governed by the generalized Korteweg-de Vries equation. Wave motion 2 (1980), 197-206.

[137] A. Soffer and W. I. Weinstein, Multichannel nonlinear scattering for nonintegrable equations. Comm. Math. Phys. 133, 116-146 (1990).

[138] A. Soffer and M. I. Weinstein, Multichannel nonlinear scattering ii. The Case of Anisotropic Potentials and Data. J. Diff. Eq. 98, 376-390 (1992).

[139] T. Tao, Nonlinear dispersive equations. Local and global analysis. CBMS Regional Conference Series in Mathematics, 106. American Mathematical Society, Providence, RI, 2006.

[140] T. Tao, Scattering for the quartic generalised Korteweg-de Vries equation, J. Diff. Eq. 232 (2007), 623-651.

[141] T. Tao, Why are solitons stable? Bull. Amer. Math. Soc. (N.S.) 46 (2009), no. 1, 1-33.

[142] M. Wadati, The modified Korteweg-de Vries Equation, J. Phys. Soc. Japan, 34, no.5, (1973), $1289-1296$.

[143] M. Wadati and K. Ohkuma, Multiple-pole solutions of modified Korteweg-de Vries equation. J. Phys. Soc. Jpn. 51 (1982), no. 6, 2029-2035.

[144] M. Wadati and M. Toda, The exact N-soliton solution of the Korteweg-de Vries equation. J. Phys. Soc. Japan 32 (1972), 1403-1411.

[145] M. I. Weinstein, Modulational stability of ground states of nonlinear Schrödinger equations. SIAM J. Math. Anal., 16 (1985), 472-491.

[146] M. I. Weinstein, Lyapunov stability of ground states of nonlinear dispersive evolution equations. Comm. Pure. Appl. Math. 39, (1986) 51-68.

[147] G. B. Whitham, Linear and Nonlinear Waves. John Wiley \& Sons Inc. 1974.

[148] J. Yang, Nonlinear waves in integrable and nonintegrable systems. Mathematical Modeling and Computation, 16. Society for Industrial and Applied Mathematics (SIAM), Philadelphia, PA, 2010.

[149] N. J. Zabusky and M. D. Kruskal, Interaction of "solitons" in a collisionless plasma and recurrence of initial states. Phys. Rev. Lett. 15 (1965), 240-243.

[150] N. J. Zakharov and M. D. Manakov, Asymptotic behavior of nonlinear wave systems integrated by the inverse scattering transform. Soviet Physics JETP 44 (1976), 106-112.

[151] V. E. Zakharov and A. B. Shabat, Exact theory of two-dimensional self-focusing and one-dimensional self-modulation of waves in nonlinear media. Soviet Physics JETP 34 (1972), 62-69.

CMls, École Polytechnique, CNRS, 91128 Palaiseau Cedex, France

E-mail address: yvan.martel@polytechnique.edu 